\title{
Particle Diffusion from the Beam-Beam Interaction in Synchrotron Colliders
}

\author{
J.K. Koga ${ }^{a)}$ and T. Tajima \\ Department of Physics and \\ Institute for Fusion Studies \\ The University of Texas at Austin \\ Austin, Texas 78712
}

\begin{abstract}
We investigate the beam-beam interaction in a synchrotron collider, specifically studying slow particle diffusion in phase space away from tune resonances. Using the tune and tune shift of contemporary large hadron colliders as reference parameters, our computation shows all particles diffusive after $10^{5}$ rotations in contrast to previous single particle tracking results. The diffusion coefficients are several orders of magnitude higher than the tracking code and increase exponentially with the action. caused by the collision induced variation of the second moment of the beams $\left\langle x^{2}\right\rangle$.
\end{abstract}

Pacs Nos.: 29.27.Bd, 52.65.+2, 29.20.Dh

\footnotetext{
${ }^{a)}$ Current address: Naka Fusion Research Establishment, Japan Atomic Energy Research Institute, Nitkit Ibaraki 311-01, Japan 
In synchrotron colliders one of the principal limitations on beam intensity is the heamlbeam interaction. ${ }^{1,2}$ In the beam-beam interaction each beam imparts an impulse on the other beam at the interaction point (IP) where the beams cross. For the hadron colliclers the beam-beam interaction is expected to be crucial, since there is little synchrotron radiation Camping to slow beam emittance growth as in electron storage rings. ${ }^{1}$

In this paper we investigate the beam-beam interaction with emphasis on subtle particle diffusion away from resonances on time-scales of the order of machine operation times $\left(\sim 10^{4}\right.$ seconds). A one dimensional model is employed at the IP so that oscillations in only one transverse direction due to the counterstreaming beams are studied. The rest of the machine is treated by symplectic harmonic transport (betatron oscillations). By employing a fully self-consistent model at the interaction point, an assessment of the relative importance of collisions as a whole and individual "soft" collisions (collective effects) can be determined. Specifically, we will examine the contribution of self-consistent effects on particle diffusion after a large number of interactions.

We briefly describe the conventional tracking and our new $\delta f$ codes used to study the beam-beam interaction. More detailed descriptions of the $\delta f$ code can be found in other references. ${ }^{3,4,5,6}$ The basic principle of conventional tracking codes is to follow the dynamics of single particles around the machine. ${ }^{7,8}$ In the beam-beam interaction the tracking code consists of two components: a target beam and a projectile beam. The target beam is treated as a rigid smooth Gaussian distribution of a large number of particles. It remains unchanged from interaction to interaction. The projectile beam is considered to be a collection of mutually noninteracting particles which are perturbed by the target beam. This is the so called "weak-strong" approximation..$^{7,8}$ In tracking code simulations in the "weak-strong" approximation, transport about one turn is simulated as the product of two matrices. (nlt for the one turn Courant-Synder map, ${ }^{9}$ and the other for the impulsive application of thr 
beam-beam interaction discussed above: ${ }^{7,8}$

$$
\begin{gathered}
{\left[\begin{array}{l}
x \\
x^{\prime}
\end{array}\right]_{\text {final }}=M\left[\begin{array}{l}
x \\
x^{\prime}
\end{array}\right]_{\text {initial }},} \\
M=\left[\begin{array}{cc}
\cos \left(2 \pi \nu_{0}\right) & \beta_{0}^{*} \sin \left(2 \pi \nu_{0}\right) \\
-\sin \left(2 \pi \nu_{0}\right) / \beta_{0}^{*} & \cos \left(2 \pi \nu_{0}\right)
\end{array}\right]\left[\begin{array}{cc}
1 & 0 \\
4 \pi \Delta \nu_{0} F(x) / 3_{0}^{*} & 1
\end{array}\right] .
\end{gathered}
$$

where $x$ is the position of the particle, $x^{\prime}$ is $d x / d s, s$ is the distance along the collirler. $\nu_{0}=\oint d s / 3(s)$ is the tune, $\Delta \nu_{0}$ is the input tune shift, $\beta_{0}^{*}$ is the betatron oscillation amplitude at the IP, and $F(x)$ is the force of a 1-D Gaussian slab:

$$
F(x)=\sqrt{\frac{\pi}{2}}\left(\frac{\sigma_{x_{0}}}{x}\right) \operatorname{erf}\left(\frac{x}{\sqrt{2} \sigma_{x_{0}}}\right),
$$

where erf is the error function and $\sigma_{x_{0}}$ is the beam standard deviation in $x$.

In a fully self-consistent "strong-strong" treatment the force $F(x)$ in Eq. (2) is solved for both beams using the Vlasov equation for the beam-beam interaction: ${ }^{10}$

$$
\left.\frac{\partial f}{\partial s}+x^{\prime} \frac{\partial f}{\partial x}-\left(K(s) x-F(x) \delta_{p}(s)\right)\right) \frac{\partial f}{\partial x^{\prime}}=0
$$

where $K(s) x$ is the magnetic guiding force, $F(x)$ is the beam kick from the other beam. and $\delta_{p}(s)$ is the periodic delta function which is nonzero at only the IP.

In previous "strong-strong" treatments the distribution $f$ was either represented by a Gaussian with varying position and width ${ }^{11}$ or by a finite number of particles. ${ }^{12}$ Each methorl has its advantages and disadvantages. While the advantage of the Gaussian treatment is the lack of finite particle fluctuation noise, the disadvantage is higher moments of the distribution do not evolve. The finite particle method allows the evolution of the higher moments, but is subject to the noise.

The $\delta f$ method substantially reduces the fluctuation noise and allows the evolution of higher moments by evolving only the perturbative part of the distribution. ${ }^{3,4,5}$ The total distribution function $f\left(x, x^{\prime}, s\right)$ is decomposed into

$$
f\left(x, x^{\prime}, s\right)=f_{0}\left(x, x^{\prime}, s\right)+\delta f\left(x, x^{\prime}, s\right)
$$


where $f_{0}\left(x, x^{\prime}, s\right)$ is the steady or slowly varying part of the distribution and $\delta f\left(x, x^{\prime} . s\right)$ is the perturbative part. For beam-beam interaction an analytic solution close to the original Vlasov equation can be found using a linearized beam-beam force whose solution is a Gaussian distribution. Equation (4) reduces to an exact form:

$$
\begin{array}{r}
\frac{\partial \delta f}{\partial s}+x^{\prime} \frac{\partial \delta f}{\partial x}-\left(K(s) x-F_{0}(x) \delta_{p}(s)\right) \frac{\partial \delta f}{\partial x^{\prime}}= \\
-\left(F(x)-F_{0} x\right) \delta_{p}(s) \frac{\partial f_{0}}{\partial x^{\prime}},
\end{array}
$$

where $F_{0}(x)$ is the kick from a Gaussian beam, $F(x)$ is the kick from a Gaussian beam $F_{0}(x)$ plus $\delta F(x)$ the force due to the perturbed distribution $\delta f$, and $F_{0}$ is the portion of the beam-beam force $F(x)$ linear in $x$. Only the nonlinear part of the beam-beam force on the right-hand side of Eq. (6) is used to advance $\delta f$. The perturbative part $\delta f\left(x, x^{\prime} . s\right)$ is then small, causes only small changes to the distribution representing the fluctuation levels. Equation (6) can be represented by $N$ particles by the method of characteristics:

$$
\delta f\left(x, x^{\prime}, s\right)=\sum_{i=1}^{N} w_{i}\left[s, x_{\mathfrak{i}}(s), x_{i}^{\prime}(s)\right] \delta\left(x-x_{\mathfrak{i}}(s)\right) \delta\left(x^{\prime}-x_{\mathfrak{i}}^{\prime}(s)\right) .
$$

where the weights of particles $w_{i}$ are evolved using Eq. (6).

We examine particle diffusion brought about by the self-consistent solution of the beambeam interaction through the $\delta f$ simulation that goes beyond the results from the "weakstrong" tracking code. We also determine the contribution from beam moments to the diffusion.

Figure 1(a) shows the diffusion coefficients of 100 randomly distributed sample particles versus their initial action calculated for the $\delta f$ code and the tracking code, where the normalized action is $J=\frac{1}{2}\left(\left(\frac{x}{\sigma_{x}}\right)^{2}+\left(\frac{p_{x}}{\sigma_{p}}\right)^{2}\right)$. The diffusion coefficients are obtained after $10^{5}$ rotations with the tune $\nu_{0}=0.285$ and tune shift $\Delta \nu_{0}=2.1 \times 10^{-3}$ similar to large hadron collider reference parameters. The diffusion coefficient $D(J)$ in the action is normalized to $\sigma_{J}^{2} / N_{r}$, where $\sigma_{J}=\sigma_{x}^{2} / \beta^{*}$ and $N_{r}$ is the number of rotations so that it takes $D(J)^{-1}$ turns to 
diffuse over one standard deviation of the beam emittance. For example. Fig l(a) shows on average the diffusion time is approximately $10^{10}$ turns in our self-consistent calculations. The distinction between the real diffusion and apparent diffusion such as phase space (moillations: is determined using the method of Chirikov where coefficients, $D_{1}$ and $D_{2}$. are calculaterl over two different timescales. ${ }^{1,2}$ If $D_{1} \approx D_{2}$, then the motion in $J$ is diffusive. If $D_{1} \gg D_{2}$ where $D_{1}$ is calculated on shorter timescales than $D_{2}$, then the motion is merely phase spact oscillations. In Fig. 1(a) the coefficients are calculated over intervals of $\Delta V_{1}=10^{3}$ and $\Delta N_{2}=10^{4}$ turns. In Fig. 1(a) the ratio of the coefficients calculated for the tracking code sample particles is on the order of $\frac{D_{2}}{D_{1}} \sim 0.01 \rightarrow 0.1$. The range of ratios indicates that the particles are showing little diffusive behavior.

We find (i) that the $D(J)$ is far greater in the $\delta f$ code than in the tracking code, indicating that conventional tracking code prediction is unrealistically low; (ii) that even the highest value of $D(J)$ from the self-consistent result remains within typical machine design lifetimes of $10^{8}$ turns; and (iii) there appears a strong action, $J$, dependence. In Fig. 1(a) for all sample particles in the $\delta f$ code the ratio $D_{2} / D_{1}$ is on the order of 1 , indicating that all particles are diffusive. The diffusion coefficient $D(J)$ is an approximately exponential function of $J$ for $J>0.5$. We also find that the alternate 'reathing oscillations of the two beams appear in the self-consistent calculations, but not in the tracking calculations. 'The onset of the oscillations is due to the collective interaction. The diffusion is not strongly dependent on the number of simulation particles. In this $\delta f$ simulation each beam has $10^{3}$ simulation particles; however. the coefficients calculated for a simulation with $N=10^{4}$ simulation particles give the same results. $^{6}$

The source of the enhanced diffusion in the self-consistent $\delta f$ simulation is identified with the observed variation of the moments of each beam which does not occur in the tracking code. The contribution of the first two beam moments $\langle x\rangle$ and $\left\langle x^{2}\right\rangle$ to the beam diffusion may be estimated by varying these moments in the tracking code which assumes a Gaussian 
beam.

When the beam moment $\langle x\rangle$ from the $\delta f$ code is input into the tracking code. the diffusion coefficients calculated for sample particles with $J<1$ are close to that of the if code. However, for $J>1$ the diffusion coefficients level off and deviate substantially from the exponentially increasing diffusion coefficients of the $\delta f$ code. Figure $1(b)$ shows diffusion coefficients from the tracking code particles when the beam $\sigma_{x}$ of the tracking code is variecl using $\left\langle x^{2}\right\rangle$ from the $\delta f$ code. The solid curve for the $\delta f$ diffusion is obtained by smoothing the diffusion coefficients calculated for 10000 rotations. The diffusion coefficients from the tracking code with the appropriate spectrum of variations of $\left\langle x^{2}\right\rangle$ and the $\delta f$ code nearly overlap for most values of $J>2$. For values of $J<2$ the tracking code coefficients are smaller than the $\delta f$ code. Thus, most of the enhanced diffusion can be accounted for by the variation of the second moment $\left\langle x^{2}\right\rangle$ incurred by collective 'breathing' modes. Diffusion at the core of the beam can be accounted for by the variations in both the first $\langle x\rangle$ and second $\left\langle x^{2}\right\rangle$ moments.

Figure 2(a) shows the frequency spectra $S_{J}(f)$ taken from the autocorrelation of $J$ for sample particles initially at $J=1,2,3$, and 4 over $M=10^{5}$ rotations. The frequency $f$ of the peak in $S_{J}(f)$ for the sample particles is decreasing with increasing $J$ and corresponds to approximately to $f=1-2\left(\nu_{0}-\Delta \nu(J)\right)$ where $\Delta \nu(J)$ is the tune shift of the particular particle. The decrease in frequency can be attributed to the decrease in $\Delta \nu(J)$ with increasing $J$ of the particle, typical of the beam-beam tune shift. Figure $2(b)$ shows the frequency spectrum $S_{\sigma_{x}}(f)$ of the second moment of motion $\sigma_{x}=\sqrt{\left\langle x^{2}\right\rangle}$ for $M=10^{5}$ rotations. The arrows indicate the upper and lower bounds of frequencies accessible to particles in the beam. The frequency $f$ of the peak in Fig. 2(b) of the $\sigma_{x}$ motion is approximately $1-2\left(\nu_{0}-\Delta \nu\right)$ where $\Delta \nu$ is the tune shift of the large $J$ particles. Sample particles with large $J$ are in resonance with the $\sigma_{x}$ variation. Sample particles with small $J$ have a characteristic frequency $f$ in their motion which is higher than the $\sigma_{x}$ frequency and are not in resonance. Therefore, the 
main contribution to the diffusion of the large $J$ particles is resonance overlap. ${ }^{1}$

Figure $1(c)$ shows the diffusion coefficients obtained from the input of $\left\langle x^{2}\right\rangle$ variation into the tracking code including only the band of frequencies $f$ shown in Fig. 2(b). There are sume particles in the range $1<J<2.2$ whose diffusion coefficients are lower than that obtained from the $\delta f$ simulation. However, the diffusion observed in the $\delta f$ code can be mostly accounted for by the variation of $\left\langle x^{2}\right\rangle$ in a narrow band of frequencies near $1-2\left(\nu_{0}-\Delta \nu^{\prime}\right)$ where $\Delta \nu$ is that for large amplitude particles.

The variation in $\langle x\rangle$ does not contribute as significantly to the particle diffusion in $J$ as $\left\langle x^{2}\right\rangle$. The characteristic frequencies of the $\langle x\rangle$ motion is not as close to the characteristic frequencies of the $J$ variation as the $\left\langle x^{2}\right\rangle$ motion.

An analytic expression for the diffusion in action $J$ can be obtained for beam $\sigma_{x}$ variation by adapting the formalism of Stupakov ${ }^{13}$ which contained external kicks with $\langle x\rangle$ variation. but no $\left\langle x^{2}\right\rangle$ variation. The change in the action due to the beam-beam kick from a one dimensional Gaussian slab can be written in the form:

$$
\Delta J=(2 \pi)^{3 / 2} \Delta \nu_{0} \sigma_{x} x^{\prime} \operatorname{erf}\left(\frac{x}{\sqrt{2} \sigma_{x}}\right),
$$

where

$$
x=\sqrt{2 J \beta} \cos (\Psi), \quad x^{\prime}=-\sqrt{\frac{2 J}{\beta}} \sin (\Psi),
$$

and $\Psi$ is the phase advance. Perturbing Eq. (8) with respect to $\sigma_{x}$ an expression of the form:

$$
\begin{gathered}
\delta(\Delta J)=8 \pi \Delta \nu_{0} J \exp \left(-\frac{J \beta}{2 \sigma_{x_{0}}^{2}}\right)\left\{I_{0}+\right. \\
\left.2 \sum_{k=1}^{\infty}(-1)^{k} I_{k} \cos [2 k \Psi]\right\} \sin [2 \Psi] \frac{\Delta \sigma_{x}}{\sigma_{x_{0}}}
\end{gathered}
$$

where $I_{k}$ represents the $k$-th modified Bessel function with arguments $\left(\frac{J \beta}{2 \sigma_{x_{0}}^{2}}\right)$. The changt in the action $J$ over $M$ turns is then after simplifying Eq. (10):

$$
\Delta J_{M}=8 \pi \Delta \nu_{0} \sum_{l=0}^{M-1} J_{l} \exp \left(-\frac{J_{l} \beta}{2 \sigma_{x_{0}}^{2}}\right) \sum_{k=0}^{\infty}\left\{I_{k}-\right.
$$




$$
\left.I_{k+2}\right\}(-1)^{k} \sin \left[2(k+1) \Psi_{l} \mid \frac{\Delta \sigma_{x}(l)}{\sigma_{x_{0}}}\right.
$$

where the arguments to the modified Bessel functions are $\left(\frac{J_{13}}{2 \sigma_{x_{0}}^{2}}\right)$. The phatse alvance is: $\Psi_{l}=2 \pi l\left(\nu_{0}+\langle\Delta \nu(J)\rangle\right)$ where $\langle\Delta \nu(J)\rangle$ is the average tune shift that the particle encumuters.

The diffusion coefficients can be calculated from:

$$
D(J)=\frac{\left\langle\Delta J_{M}^{2}\right\rangle}{M}
$$

where

$$
\begin{array}{r}
\Delta J_{M}^{2}=64 \pi^{2} \Delta \nu_{0}^{2} J^{2} \exp \left(-\frac{J \beta}{\sigma_{x_{0}}^{2}}\right) \sum_{l, m=0}^{M-1} \frac{\Delta \sigma_{x}(l)}{\sigma_{x_{0}}} \frac{\Delta \sigma_{x}(m)}{\sigma_{x_{0}}} \times \\
\sum_{k, r=0}^{\infty}\left\{I_{k}-I_{k+2}\right\}\left\{I_{r}-I_{r+2}\right\}(-1)^{k+r} \sin \left[2(k+1) \Psi_{l}\right] \sin \left[2(r+1) \Psi_{m}\right] .
\end{array}
$$

In Eq. (13) the action, $J$, is assumed not to vary much over the $M$ turns. Equation (13) can be further simplified by rearranging terms:

$$
\begin{array}{r}
\Delta J_{M}^{2}=32 \pi^{2} \Delta \nu_{0}^{2} J^{2} \exp \left(-\frac{J \beta}{\sigma_{x_{0}}^{2}}\right) \times \\
\sum_{n=-\infty}^{\infty} K(n) \sum_{k=0}^{\infty}\left(I_{k}-I_{k+2}\right)^{2} \cos \left[2(k+1) \Psi_{n}\right]
\end{array}
$$

where $K(n)$ is the correlation function of the $\sigma_{x}$ variations over turn $n$ :

$$
K(n)=\sum_{m=0}^{M-1} \frac{\Delta \sigma_{x}(m)}{\sigma_{x_{0}}} \frac{\Delta \sigma_{x}(m+n)}{\sigma_{x_{0}}} / M
$$

The correlation function may be defined in terms of the power spectrum, $S_{\sigma_{x}}\left(\omega^{\prime}\right)$ :

$$
S_{\sigma_{x}}(\omega)=\sum_{n=1}^{M} K(n) \exp (i \omega n)
$$

Using this expression in Eq. (14) and substituting into Eq. (12) we get

$$
D(J)=32 \pi^{3} \Delta \nu_{0}^{2} J^{2} \exp \left(-\frac{J \beta}{\sigma_{x_{0}}^{2}}\right) \sum_{k=0}^{\infty}\left(I_{k}-I_{k+2}\right)^{2} S_{\sigma_{x}}\left(\omega_{k}\right),
$$


where $\omega_{k}=2(k+1) 2 \pi\left(\nu_{0}+\langle\Delta \nu(J)\rangle\right)$. The peak in the power spectra $S_{\sigma_{x}}(f)$ in Fig. 2(h) is at $4 \pi \nu_{0}$ which corresponds to $k=0$ in Eq. (12). Figure $1(\mathrm{~d})$ shows the diffusion obtainerl from Eq. (12) for $k=0$. The power spectra in Fig. 2(b) is used where the values at a particular action $J$ is obtained from measuring the frequency of the peaks in the power spectra of the sample particle motion (Fig. 2(a)). Reasonable agreement between the $\delta f$ computation and this analytic expression is found. If we used naive approximations for the correlation function such as the Lorentzian, we were unable to reproduce the exponential $J$ depenclence of $D(J)$ for $J>0.5$.

In summary, through our extensive computation and theory we have discovered that the diffusion obtained from the self-consistent $\delta f$ code is several orders of magnitude higher than that of the prediction from conventional tracking codes. The essence of the culprit of this enhanced diffusion is captured by the variation of the second moment of the beams $\left\langle x^{2}\right\rangle$ which is the result of beam-beam interaction induced collective variations of the beam distribution.

This work was supported by the U.S. Department of Energy, Texas National Research Lab. Commission, and SSCL. 


\section{References}

${ }^{1}$ B. V. Chirikov, J. Ford, and F. Vivaldi, in Nonlinear Dynamics und the Betuln-Betum Interaction, AIP Conf. Proc. 57, edited by M. Month and J. C. Herrera (AIP. New York. 1979).

${ }^{2} \mathrm{~T}$. Bountis and S. Tompaidis, Institute for Fusion Studies Report 434. University of 'Texis. 1990 (unpublished).

${ }^{3}$ T. Tajima and F. W. Perkins, in Proc. 1983 Sherwood Theory Meeting (University of Maryland, Arlington, VA, 1983).

${ }^{4}$ M. Kotschenreuther, Bull. Am. Phys. Soc. 33, 2109 (1988).

${ }^{5}$ G. DiPeso, E. C. Morse, and R. W. Ziolkowski, J. Comput. Phys. 96, 325 (1991).

${ }^{6}$ J. K. Koga and T. Tajima, submitted to J. of Comp. Phys. (1993).

'D. Neuffer, A. Riddiford, and A. G. Ruggiero, IEEE Trans. Nucl. Sci. NS-30, 2430 (1983).

${ }^{8}$ A. G. Ruggiero, Part. Accel. 19, 157 (1986).

${ }^{9}$ E. D. Courant and H. S. Synder, Ann. Phys. 3, 1 (1958).

${ }^{10}$ A. W. Chao, SSCL-346 (1991).

${ }^{11} \mathrm{G}$. Jackson and R. H. Siemann, Nucl. Inst. and Methods 286, 17 (1990).

${ }^{12}$ S. Krishnagopal and R. Siemann, Phys. Rev. Lett. 67, 2461 (1991).

${ }^{13}$ G. Stupakov, SSCL-560 (1991). 


\section{Figure Captions}

1. $D(J)$ from (a) our $\delta f$ code and conventional tracking code, (b) tracking code pirticles with variations in $\left\langle x^{2}\right\rangle$ input from the $\delta f$, and (c) tracking code particles with $v^{2}$. input using a band of frequencies around $2\left(\nu_{0}-\Delta \nu\right)$ for $M=10^{5}$ rotations. $D_{1}$ and $D_{2}$ have time scales of $\Delta N_{1}=1000$ and $\Delta N_{2}=10000$ rotations respectively. (1) The diffusion $D(J)$ from $\delta f$ and our analytic theory.

2. After $M=10^{5}$ rotations (a) part of the frequency spectra of sample particles from the tracking code initially at $J=1,2,3$, and 4 and (b) of $\sigma_{x}$ from the $\delta f$ simulation where the arrows indicate the range of frequencies with and without the tune shift. 

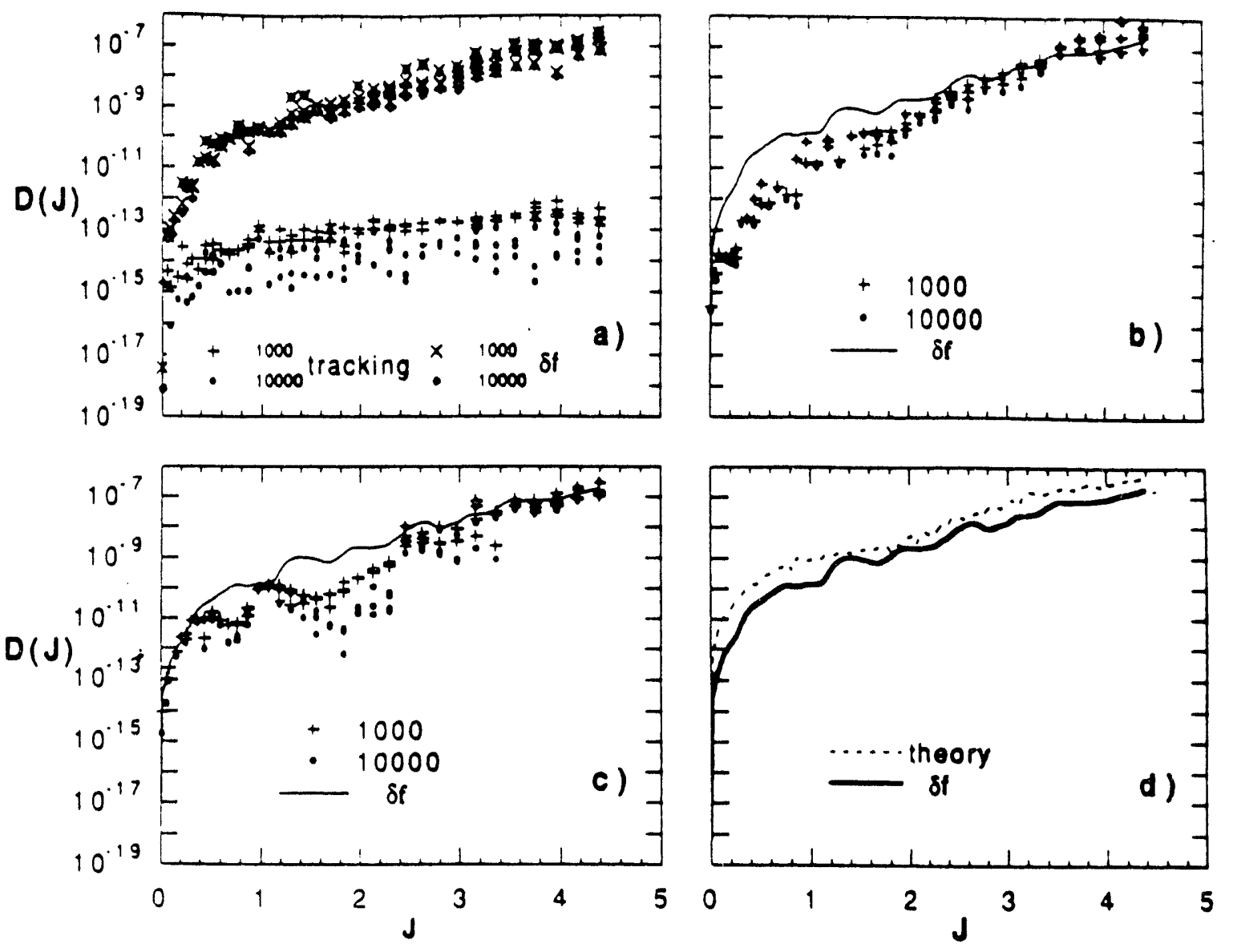

Fig. 1 


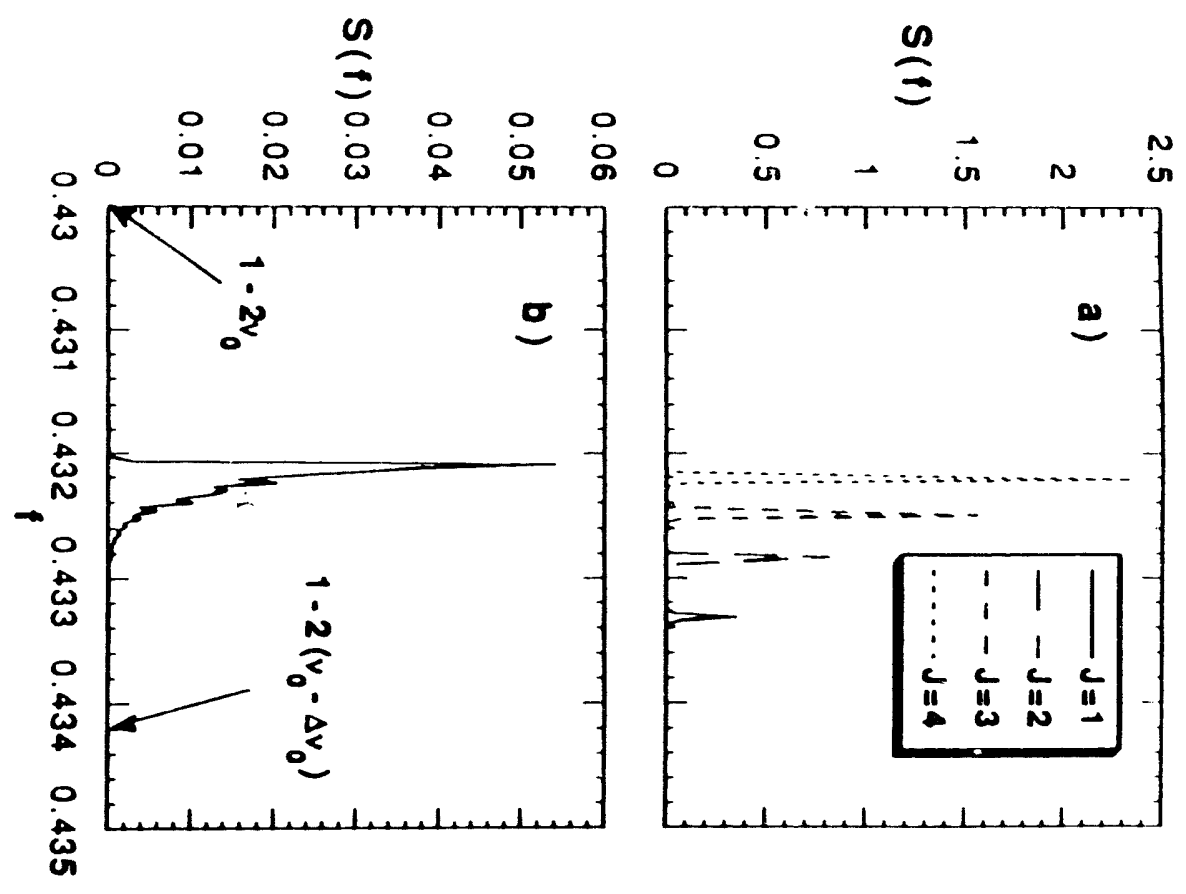



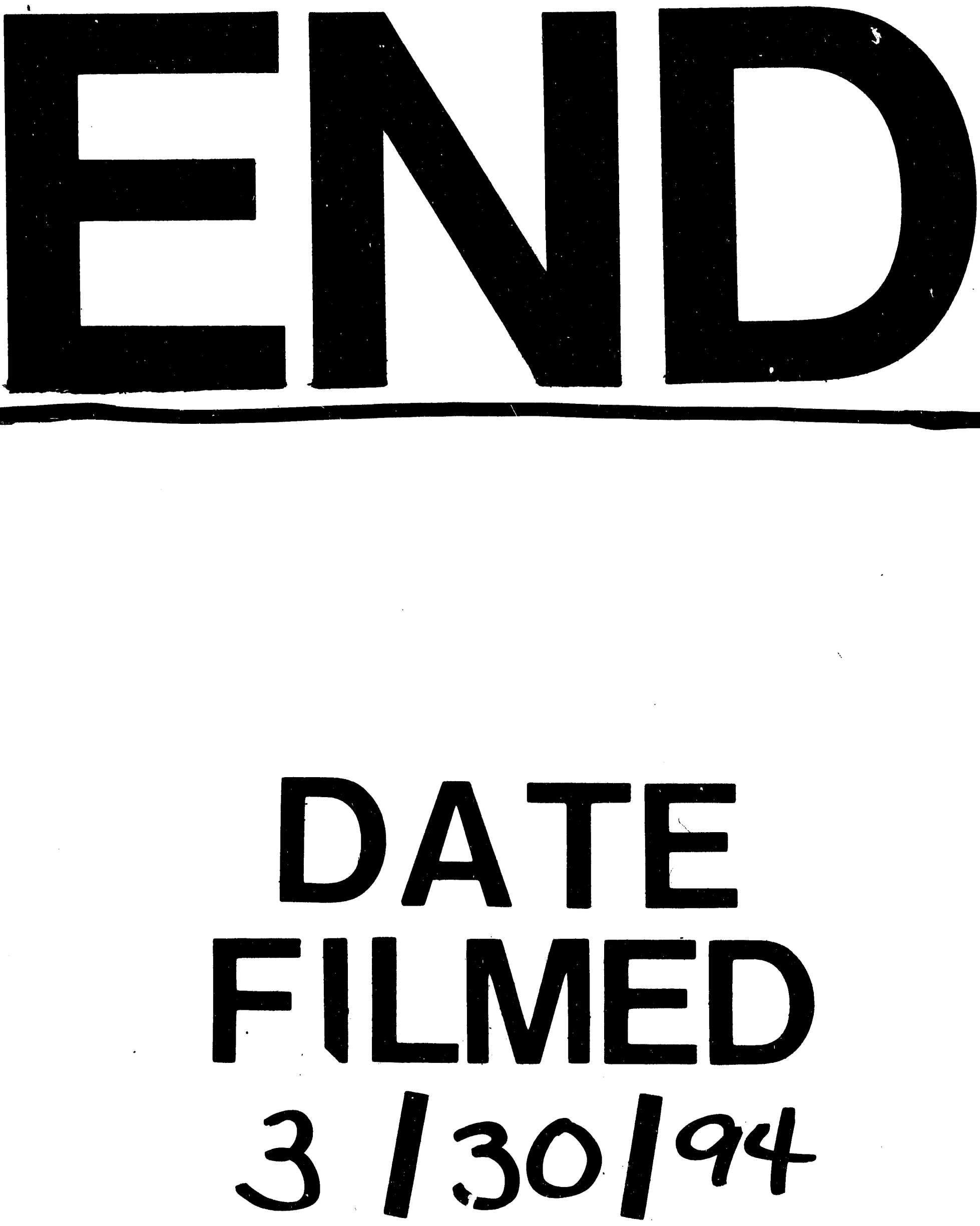
